El pasado de Palestina en disputa: Arqueología y religión en el conflicto palestino-israelí

Emanuel Pfoh

páginas / año 8 - n 18 Septiembre - Diciembre / ISSN 1851-992X/ pp.10-24 /

2016

http://revistapaginas.unr.edu.ar/index.php/RevPaginas

\title{
El pasado de Palestina en disputa: Arqueología y religión en el conflicto palestino-israelí
}

\author{
Disputing Palestine's Past: \\ Archaeology and Religion in the Palestinian-Israeli Conflict
}

\author{
Emanuel Pfoh \\ Universidad Nacional de La Plata \\ Instituto Multidisciplinario de Historia y Ciencias Humanas \\ Consejo Nacional de Investigaciones Científicas y Técnicas, Argentina \\ epfoh@conicet.gov.ar
}

\begin{abstract}
Resumen
El presente artículo aborda temáticas centrales acerca de la composición cultural e histórica del moderno conflicto palestino-israelí, en particular la relevancia del pasado más antiguo de la región de Palestina para distintos actores a través del tiempo. Cuestiones relativas a la identidad cultural de Occidente en relación con Palestina, a la arqueología bíblica, a la aparición del sionismo como movimiento nacionalista, al rol de la arqueología en el Estado de Israel y al desarrollo de una arqueología palestina de la región son tratados, si bien sumariamente, en su interrelación para comprender las variadas raíces del conflicto desde una perspectiva histórica.
\end{abstract}

\section{Palabras Clave}

Israel; Palestina; conflicto; arqueología; Biblia; sionismo

\begin{abstract}
This paper addresses key thematic issues relating to cultural and historical aspects of the modern Palestinian-Israeli conflict, especially in regard to the relevance of Palestine's ancient past for different historical actors. Questions related to the cultural identity of the West and Palestine, biblical archaeology, Zionism and nationalism, the role of archaeology in the State of Israel and the development of a Palestinian archaeology are concisely treated in their interrelationship in order to expose an historical understanding of the conflict.
\end{abstract}

\section{Keywords}

Israel; Palestine; conflict; archaeology; Bible; Zionism

Esta obra está sujeta a la Licencia Reconocimiento-NoComercial-CompartirIgual 4.0 Internacional de Creative Commons. http://creativecommons.org/licenses/by-nc-sa/4.0/ 


\section{Emanuel Pfoh}

\section{Palestina y la identidad cultural del Occidente cristiano}

El territorio de Palestina-entendido en términos generales como el espacio geográfico que se encuentra entre el río Litani y los Altos del Golán al norte, el río Jordán al este y el desierto del Sinaí al sudoeste, aunque sus fronteras y límites han variado de diverso modo a lo largo de los siglos - ha tenido durante dos milenios un rol de considerable importancia para el mundo occidental por ser el escenario, primeramente, de los relatos de la Biblia Hebrea (o del Antiguo Testamento), y luego, de los inicios mismos del cristianismo; instancias inaugurales, junto con los componentes culturales de Grecia y Roma, de la civilización de Occidente. En efecto, ya los Padres de la Iglesia concibieron a Palestina como terra sancta, como un espacio de singular importancia ontológica, puesto que fue allí mismo, y no en otro lugar de la tierra, que se produjo la revelación, el ministerio y la resurrección de Jesucristo ${ }^{1}$.

Esta comprensión de Palestina como lugar fundacional durante los primeros siglos del cristianismo bien podría entenderse como una primera apropiación simbólica del territorio-no obstante el imaginario de las poblaciones nativas- desde centros políticos y culturales externos. Tal apropiación, refrendada especialmente por la conversión al cristianismo del emperador Constantino I El Grande (324-337), junto con su manifestación material, no hizo sino engrosar una serie de instancias consecutivas de apropiación del Levante meridional por parte de fuerzas invasoras que se remonta hasta mediados del segundo milenio a.C. ${ }^{2}$ La ocupación del imperio bizantino fue sucedida por la conquista por parte de los ejércitos islámicos del califa Umar ibn al-Khattab (634-644) en 636-638.Las cruzadas europeas a Tierra Santa recuperaron el territorio de Siria-Palestina para el cristianismo por casi dos siglos (1099-1291)33, hasta que nuevamente su posesión se repartió entre distintos poderes externos islámicos: los ayubíes, desde la conquista de Jerusalén por Saladino en 1187hasta 1260;los mongoles, en distintas incursiones en el Levante durante las segunda mitad del siglo XIII; los mamelucos de Egipto, hasta 1516 y, finalmente, en ese mismo año, los otomanos, hasta 1918, en el contexto de la Primera Guerra Mundial (1914-1918). La corona británica se haría luego cargo del

\footnotetext{
1Véase R.L. Wilken. The Land Called Holy: Palestine in Christian History \& Thought. New Haven, Yale University Press, 1992; E.H. Tejirian y R. Spector Simon. Conflict, Conquest, and Conversion: Two Thousand Years of Christian Missions in the Middle East. Nueva York, Columbia University Press, 2012. 2 Palestina, debido a su ubicación estratégica entre continentes, fue ocupada por varios poderes a través de milenios. En primer lugar, por el imperio egipcio durante unos tres siglos (desde los inicios del siglo XV hasta comienzos del siglo XII a.C., aproximadamente). Luego de un período transicional, del cual surgen organizaciones políticas autónomas (entre otras, el reino de Israel, entre 900 y 722 a.C.), la región fue nuevamente ocupada, con breves lapsos de autonomía local, por fuerzas asirias, babilónicas, persas, helenísticas y romanas hasta la llegada de la hegemonía romanooriental/bizantina a la que referimos.

${ }^{3}$ La presencia cruzada en Siria-Palestina puede dividirse en dos períodos: 1099-1187, con el Primer Reino de Jerusalén, y 1192-1291, con el Segundo Reino de Jerusalén o Reino de Acre; cf. C. Tyerman. God's War: A New History of the Crusades. Londres, Penguin Books, 2007, esp. pp. 176-240, 354-374 y 715-822.
} 


\section{El pasado de Palestina en disputa: Arqueología y religión en el conflicto palestino-israelí}

control militar y de la administración formal de Palestina hasta las vísperas de la proclamación del Estado de Israel en 19484.

En plena ocupación otomana del territorio, durante la célebre expedición de Napoleón Bonaparte a Egipto de 1798-1801 y siete siglos después de la Primera Cruzada, Europa habría de incursionar nuevamente en Palestina en 1799. Este año, en verdad, puede considerarse como el hito que marca el redescubrimiento de Palestina por parte del Occidente cristiano, pero, a partir de ahora, esencialmente bajo coordenadas de exploración científica. Esta nueva apropiación simbólica del paisaje histórico, estaba conducida por una visión racional, representada por disciplinas como la topografía, la geología, la arqueología, etc.; no obstante, un impulso sociocultural cristiano (notablemente, evangélico-protestante) y el deseo por recuperar paisajes bíblicos hicieron las veces de primer motor detrás de varias de estas investigaciones ${ }^{5}$.

\section{Investigaciones en arqueología bíblica (1865-1948)}

Desde tiempos del emperador Constantino, Palestina no dejó de recibir peregrinos, tanto judíos como cristianos y posteriormente musulmanes (aunque de manera intermitente, de acuerdo con las situaciones políticas que atravesaba la región), y de ese modo, especialmente a partir de tiempos medievales, se fue gestando un acervo de conocimiento y representación occidental de la geografía sagrada de la región que se mantuvo hasta los inicios de la era moderna ${ }^{6}$. Con la mencionada expedición napoleónica, al peregrinaje y a la exploración geográfica y religiosa de Palestina, se integró-decíamos- la intención de comprender científicamente la historicidad del pasado bíblico, de transitar por caminos y habitar locaciones anteriormente transitadas y habitadas por personajes bíblicos, lo cual propició y condujo en gran medida la investigación sistemática del territorio en sus diversos aspectos, desde la historia natural hasta la producción cultural de las sociedades que lo ocuparon. Si bien dichas exploraciones comienzan de manera

\footnotetext{
4 Cf. B. Scarcia Amoretti. "La Palestine musulmane", en A. Giardina, M. Liverani y B. Scarcia Amoretti.La Palestine: Histoire d'une terre, (Comprendre le Moyen Orient).París, L'Harmattan, 1990, pp. 117-208; G. Krämer.Historia de Palestina: Desde la conquista otomana hasta la fundación del Estado de Israel. Madrid, Siglo XXI, 2006 [edición original en alemán, 2002].

5Véase P.R.S. Moorey.A Century of Biblical Archaeology. Cambridge, Lutterworth Press, 1991; J.J. Moscrop.Measuring Jerusalem: The Palestine Exploration Fund and British Interest in the Holy Land.Leicester, Leicester University Press, 2000; H. Goren.»Zieht und erforscht das Land «. Die deutsche Palästinaforschung im 19. Jahrhundert. Gotinga, Wallstein, 2003; idem.Dead Sea Level: Science, Exploration and Imperial Interests in the Near East. Londres, I.B. Tauris, 2011.Cf. también G. Biger.The Boundaries of Modern Palestine, 1840-1947.Londres, RoutledgeCurzon, 2004.

6Véase H. Donner.Pilgefahrt ins Heilige Land: Die ältesten Berichte christlicher Palästinapilger (4.-7. Jh.). Stuttgart, Verlag Katholisches Bibelwerk 2002; S. Lehmann-Brauns.Jerusalem sehen. Reiseberichte der 12. und 15. Jahrhunderts als empirische Anleitung zur geistigen Pilgefahrt, (Berliner Kulturwissenschaft, 9). Freiburg im Breisgau, Rombach Verlag, 2010; V. Türck.Christliche Pilgerfahrten nach Jerusalem im früheren Mittelalter im Spiegel der Pilgerbericht, (ADPV, 40).Wiesbaden, Harrassowitz, 2011; S. Sand.The Invention of the Land of Israel: From Holy Land to Homeland. Londres, Verso Books, 2012, pp. 119-175.
} 


\section{Emanuel Pfoh}

irregular con las aventuras e incursiones de algunos individuos notables en las primeras décadas del siglo XIX, la fundación en Londres del Palestine Exploration Fund en 1865-al que luego se le sumará en Alemania el Deutscher Verein zur Erforschung Palästinas en 1877-, marca el inicio formal e institucional de esta indagación científica sobre Palestina, actividad coronada con una serie de relevamientos topográficos y científicos pioneros: la "Survey of Western Palestine" (1871-1877), la "Survey of Eastern Palestine" (1883-1884) y la "Survey of the Wilderness of Zin" (1913)7.

A la par de la investigación científica, Palestina fue igualmente comprendida durante este período en claros términos geopolíticos y de valor estratégicoeconómico en Medio Oriente por parte del gobierno británico, y también de otros poderes europeos ${ }^{8}$. Así pues, luego de la caída del imperio otomano en 1918, Gran Bretaña ocupó no sólo Palestina sino también las regiones de Transjordania e Irak (los británicos habían ocupado también Egipto en 1882), bajo el esquema de protectorado a través de un Mandato sobre estos territorios (1920-1948). Dicha ocupación territorial fue posible, en gran medida, gracias a las actividades de inteligencia militar llevadas a cabo en conjunto con las exploraciones y relevamientos científicos inmediatamente anteriores ${ }^{9}$.

Ya durante el Mandato británico, las exploraciones arqueológicas y geográficas de Palestina reproducían la unión entre el antiguo pasado bíblico y el presente de un Occidente cristiano que reclamaba el territorio como suyo, no solamente en términos geopolíticos sino también —como ya indicamossimbólicos, como parte fundacional de la cultura occidental. Es, por cierto, durante estas primeras décadas del siglo XX que dicho reclamo de pertenencia territorial experimentó una transición de agente principal: del Occidente cristiano al movimiento sionista. En efecto, y de modo análogo a la imaginación y la representación post-napoleónica del territorio, el sionismo europeo imaginó a Palestina a partir de la construcción de una geografía cultural del territorio anclada en una interpretación secular de las tradiciones bíblicas y en ciertas reivindicaciones sociales y políticas. Los pobladores árabes palestinos, especialmente los habitantes de aldeas y campesinos (fellahin) y los beduinos, fueron inicialmente invisibilizados por esta concepción victoriana compartida por científicos europeos, colonos evangélicos e ideólogos sionistas; su presencia, durante las primeras décadas del siglo $\mathrm{XX}$, tampoco fue tomada demasiado en

\footnotetext{
${ }^{7}$ Cf. Moscrop, Measuring Jerusalem..., Op. Cit.; Goren, »Zieht und erforscht das Land«..., Op. Cit., pp. 320-339; Goren, Dead Sea Level..., Op. Cit., pp. 1-115.

${ }^{8}$ N.A. Silberman. Digging for God and Country: Exploration, Archaeology, and the Secret Struggle for the Holy Land, 1799-1917.Nueva York, Doubleday, 1982; H. Goren. "Scientific Organizations as Agents of Change: The Palestine Exploration Fund, the Deutsche Verein zur Erforschung Palästinas and Nineteenth-Century Palestine". Journal of Historical Geography, vol. 27, 2001, pp. 153-165; D. Trimbur y R. Aaronsohn, (editores).De Bonaparte à Balfour: La France, l'Europe occidentale et la Palestine, 1799-1917.París, CNRS Éditions, 2008 [2001].

${ }^{9}$ J. Barr. A Line in the Sand: Britain, France and the Struggle that Shaped the Middle East. Londres, Simon\&Schuster, 2011; sobre el mapeo de Palestina en este período, cf. D. Gavish. A Survey of Palestine under the British Mandate, 1920-1948. Londres, RoutledgeCurzon, 2005.
} 


\section{El pasado de Palestina en disputa: Arqueología y religión en el conflicto palestino-israelí}

cuenta, excepto como ejemplo vivo de un pasado bíblico evocado originalmente desde la religiosidad y la tradición pero ahora superado por el progreso y la modernización que se arrogaba el sionismo en esa nueva tierra prometida ${ }^{10}$.

\section{Sionismo y nacionalismo}

El siglo XIX fue el período en el que se configuraron los nacionalismos modernos en Europa. Y, por cierto, no resulta extraño que durante la primera mitad de ese siglo autores alemanes de origen judío, como I.M. Jost (1793-1860), L. Zunz (1794-1886), M. Hess (1812-1875) y H. Graetz (1817-1891), comenzaran a escribir historias nacionales del pueblo judío, de manera similar a las historias nacionales del pueblo alemán o del pueblo francés publicadascontemporáneamente ${ }^{11}$. Es con las obras de estos intelectuales que la idea de "pueblo judío" dejó de remitir primariamente a una confesión de fey pasó a denotar una categoría nacional, que incorporaba a aquella fe, pero que emplazaba a su práctica en un lugar secundario y que la trascendía en tanto que una comunidad nacional, una nación judía comenzaba a ser imaginada, se profesara la fe o no. Este protonacionalismo judío de la primera mitad del siglo XIX fue el antecesor directo del sionismo político que se desarrolló durante la segunda mitad de dicho siglo ${ }^{12}$. En efecto, Shlomo Sand refiere a este mismo proceso como "la invención del pueblo judío" a partir de coordenadas seculares y no religiosas ${ }^{13}$. Y también, con la invención nacionalista del pueblo judío, señala Sand, se produjo una "invención de la tierra de Israel" como territorio nacional: eretz Israel, un concepto ambiguo en sus alcances territoriales específicos, empleado en las escrituras bíblicas y rabínicas para referirse grosso modo a Palestina y, en especial, a Jerusalén y a la zona de Judea como "territorio espiritual" del judaísmo, adoptó una nueva dimensión hegemónica, con la prédica y la expansión del sionismo, como territorio nacional ${ }^{14}$.

Esta nueva comprensión de Palestina tuvo su mayor expresión en la representación misma del territorio. Con el inicio de la colonización sionista en 1882y una presencia minoritaria de colonos judíos en términos absolutos, pero que aumentaba a medida pasaban las décadas, se hizo necesaria una nueva manera de concebir el territorio acorde con el crecimiento demográfico judío y la ideología

\footnotetext{
${ }^{10}$ Véase en mayor detalle E. Pfoh.“Geografías imaginadas, práctica arqueológica y construcción nacional en Israel/Palestina”. Cuadernos de Antropología Social, vol. 39, 2014, pp. 39-62; y G. Piterberg. The Returns of Zionism: Myth, Politics and Scholarship in Israel. Londres, Verso Books, 2008, pp. 192-208.

${ }^{11} \mathrm{Cf}$. S. Sand. The Invention of the Jewish People. Londres, Verso Books, 2009, pp. 87-107.

${ }^{12}$ Adoptamos aquí la diferenciación entre "sionismo práctico" y "sionismo político" que realiza Krämer, Historia de Palestina..., Op. Cit., pp. 97-115.

${ }^{13}$ Sand. The Invention of the Jewish People..., Op. Cit.

${ }^{14}$ Véase Sand.The Invention of the Land of Israel...,Op. Cit.,pp. 177-253.En particular, sobre Jerusalén, cf. N. Abu el-Haj.Facts on the Ground: Archaeological Practice and Territorial Self-Fashioning in Israeli Society. Chicago, The University of Chicago Press, 2001, pp. 130-238; también M. Hawari. "The Citadel of Jerusalem: A Case Study in the Appropriation of Archaeology in Palestine". Present Pasts, vol. 2, 2010, pp. 89-95.
} 


\section{Emanuel Pfoh}

política del sionismo, que lo reclamaba como propio. Las investigaciones topográficas del Palestine Exploration Fund habían sentado ya las bases para que posteriormente, a partir de 1922 la Jewish Palestine Exploration Society comenzara a producir mapas de Palestina con una toponimia desarabizada, reemplazando los nombres en árabe de accidentes geográficos y de poblados $\mathrm{y}$ aldeas con una nomenclatura hebraizada, "restituyendo", en palabras de los especialistas sionistas, los nombres originales (i.e., bíblicos) del paisaje natural y cultural de lo que ahora era comprendido como la tierra de Israel ${ }^{15}$. Esta reconfiguración cartográfica de Palestina fue posteriormente mantenida y desarrollada durante las primeras décadas de existencia del Estado de Israel.

\section{El Estado de Israel y la arqueología bíblica}

Con el antecedente de la exploración geográfica de Palestina en el siglo XIX, la investigación arqueológica israelí tuvo su matriz conceptual en la arqueología bíblica europea y norteamericana. A partir de la fundación del Estado de Israel en 1948, la arqueología israelí mantuvo como propias dichas tradiciones científicas dominantes, arribando a conclusiones con una fuerte impronta nacionalista. La concepción, por ejemplo, de parte de los arqueólogos israelíes de las primeras dos décadas del Estado, de la cultura material de la Edad del Bronce (ca. 3300-1200 a.C.) como propia de un "período cananeo" en la zona, reemplazada luego por la cultura material de la Edad del Hierro (ca. 1200-600 a.C.), exponiendo un "período israelita" en la región, pone de relieve el sesgo nacionalista y étnico que dominaba la labor de interpretación arqueológica, y que expresaba un traspaso de hegemonía cultural y "nacional" en el registro arqueológico de la Palestina pre-helenística, reflejando asimismo la transición hacia una hegemonía política israelí producida a partir de 1948. La interpretación del pasado se ponía así al servicio del presente para demostrar la legitimidad del Estado de Israel sobre el territorio que había comenzado a ocupar institucional y militarmente, y también con la intención de exponer las raíces más antiguas de la presencia israelita y, por lo tanto, judía en el registro arqueológico de Palestina ${ }^{16}$.

La Guerra de los Seis Días en Junio de 1967, que había tenido como resultado la conquista de Jerusalén oriental por parte de Israel, además de los territorios ahora formalmente llamados Judea y Samaria (la Ribera Occidental), entre otros, abrió la

\footnotetext{
${ }^{15}$ Cf. M. Benvenisti. Sacred Landscape: The Buried History of the Holy Land since 1948.Berkeley, University of California Press, 2000, pp. 11-54; M. Azaryahu y A. Golan. "(Re)Naming the Landscape: The Formation of the Hebrew Map of Israel 1949-1960".Journal of Historical Geography, vol. 27, 2001, pp. 178-195; B. Ra'ad. Hidden Histories: Palestine and the Eastern Mediterranean. Londres, Pluto Press, 2010, pp. 175-195.

${ }^{16} \mathrm{Cf}$. Abu el-Haj. Facts on the Ground..., Op. Cit., pp. 105-111; R. Arav. "Archaeology in the Service of Ideology in Israel", en L.J. Greenspon y R.A. Simkins (eds.), "A Land Flowing with Milk and Honey": Visions of Israel from Biblical to Modern Times.Omaha, Creighton University Press, 2001, pp. 95-104; N.P. Lemche. "History as an Argument for Land Possession", en E. Pfoh y K.W. Whitelam, (editores).The Politics of Israel's Past: The Bible, Archaeology and Nation-Building, (SWBA, 2/8). Sheffield, Sheffield Phoenix Press, 2013, pp. 102-119.
} 


\section{El pasado de Palestina en disputa: Arqueología y religión en el conflicto palestino-israelí}

oportunidad para expandir espacialmente la exploración arqueológica. En efecto, a partir de 1967, y en particular durante las dos décadas siguientes, se llevaron a cabo una serie de prospecciones arqueológicas de superficie (surveys) que permitieron componer, eventualmente, una nueva explicación sobre los orígenes "nativos" de los antiguos israelitas en Palestina hacia fines del segundo milenio a.C., contrariamente a lo que podía interpretarse en el texto bíblico ${ }^{17}$. Este desarrollo coincidió, en efecto, con la progresiva profesionalización de la arqueología israelí, cada vez menos absorbida por sentimientos nacionalistas y por intenciones de recuperar el pasado bíblico, como en décadas anteriores.

Por otro lado, y especialmente durante los años '70, el nacionalismo israelí, otrora secular en todas sus manifestaciones, fue adquiriendo tintes religiosos, inclusive ortodoxos y fundamentalistas, en ciertos sectores de la sociedad, que reclamaban -y que todavía lo hacen- la ocupación total del Gran Israel bíblico, el Israel de los reyes David y Salomón, desde el río Éufrates en Siria hasta la península de Sinaí y algunos territorios transjordanos ${ }^{18}$. Desde esta última perspectiva fundamentalista, la Biblia se transformaba en un documento de propiedad de la tierra otorgada por Dios, y todos aquellos que no estaban incluidos en dicho plan divino -notablemente, los palestinos- debían ser expulsados de la tierra de Israel. Algunos de estos grupos fundamentalistas, en particular el Gush Emunim (el "bloque de los fieles") en la Ribera Occidental, utilizaron la arqueología y sus resultados como testimonio material de la presencia milenaria del pueblo judío en Palestina y como confirmación de sus derechos a recuperar un territorio concedido por la divinidad y que debía ser redimido de la población no judía que lo ocupó hasta el momento del retorno del pueblo judío a eretz Israel $^{19}$. Este mismo entendimiento se verifica igualmente en el presente, aunque con algunas variaciones, en la fundamentación del financiamiento privado de excavaciones arqueológicas en la sección oriental de Jerusalén ${ }^{20}$.

\footnotetext{
17Véase, principalmente, I. Finkelstein.The Archaeology of the Israelite Settlement. Jerusalén, Israel Exploration Society, 1988, esp. pp. 34-117. Sobre la relevancia de las surveys para una nueva comprensión de la historia pre-helenística de Palestina, cf. T.L. Thompson. Early History of the Israelite Peoples: From the Written and Archaeological Sources, (SHANE, 4). Leiden, E.J. Brill, 1992, pp. 127-170.

18 Cf. N. Masalha. Imperial Israel and the Palestinians: The Politics of Expansion. Londres, Pluto Press, 2000, pp. 105-162.

${ }^{19}$ Véase R. Kark. "Land-God-Man: Concepts of Land Ownership in Traditional Cultures in Eretz-Israel”, en A.R.H. Baker y G. Biger, (editores).Ideology and Landscape in Historical Perspective. Cambridge, Cambridge University Press, 1992, pp. 63-82, esp. 69-78; M. Feige. "Recovering Authenticity: WestBank Settlers and the Second Stage of National Archaeology", en P.L. Kohl, M. Kozelsky y N. BenYehuda, (editores).Selective Remembrances: Archaeology in the Construction, Commemoration, and Consecration of National Pasts. Chicago, University of Chicago Press, 2007, pp. 277-298. En particular sobre el fundamentalismo judío, cf. I. Shahak y N. Mezvinsky. Jewish Fundamentalism in Israel.Londres, Pluto Press, 2004; sobre los Gush Emunim, pp. 78-95.

20 Cf. R. Greenberg. “Extreme Exposure: Archaeology in Jerusalem, 1967-2007".Conservation and Management of Archaeological Sites, vol. 11, 2009, pp. 262-281.
} 


\section{Emanuel Pfoh}

\section{El desarrollo de una arqueología palestina y la "nueva arqueología bíblica”}

A partir de la segunda mitad del siglo XX, cuando el nacionalismo palestino se hubo constituido formalmente ya como un elemento de incidencia en la política y la sociedad israelíes ${ }^{21}$, una arqueología propiamente palestina comenzó a desarrollarse de manera concreta. Al respecto, se debe tener en cuenta, por ejemplo, el notable antecedente en los estudios sobre el folklore y la etnografía palestinos que significó la obra de Tawfīq Canaan (1882-1964) durante las primeras cuatro décadas del siglo $\mathrm{XX}^{22}$.Sin embargo, la arqueología científica de Palestina por parte de palestinos inicia recién en los años '70, especialmente en la Universidad de Birzeit y bajo la dirección de Albert Glock, arqueólogo norteamericano instalado desde entonces en Israel/Palestina. Esta primera fase, de organización y formación de recursos humanos especializados, se extendió hasta principios de los años '90, coincidentemente con la muerte bajo circunstancias extrañas de Glock en 1992. A partir de ese momento, y si bien en 1994 la flamante Autoridad Nacional Palestina constituyó el Departamento Palestino de Antigüedades y Patrimonio Cultural, la arqueología de Palestina hecha por palestinos estuvo jalonada por disputas internas en diversos ámbitos políticos del movimiento nacionalista palestino,algunos de cuyos miembros, por ejemplo, intentaban demostrar la antigüedad de los palestinos en la región a través de la creación (o la exposición) de vínculos con los antiguos cananeos de la Edad del Bronce. Ésta era, en rigor, una estrategia similar a aquella utilizada por los arqueólogos israelíes durante las primeras décadas del Estado, apelando a la cultura material de los antiguos israelitas. Dicha politización de la arqueología palestina no ayudó en nada a su sustanciación como disciplina

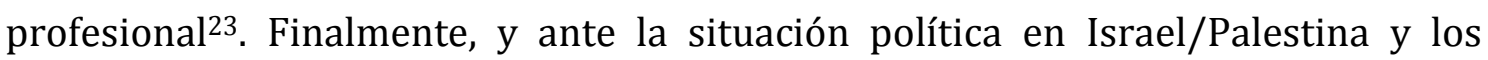
obstáculos que la ocupación israelí de la Ribera Occidental generaba para el flujo normal de recursos materiales y humanos, el Departamento de Arqueología de la Universidad de Birzeit cerró sus puertas en 2003, aunque actualmente la actividad

\footnotetext{
${ }^{21}$ Sobre la conformación del nacionalismo palestino, cf. R. Khalidi. Palestinian Identity: The Construction of Modern National Consciousness. Nueva York, Columbia University Press, 1997.

22 Véase B. Mershen y U. Hübner. "Tawfiq Canaan and His Contribution to the Ethnography of Palestine", en U. Hübner (editor).Palaestina exploranda. Studien zur Erforschung Palästinas im 19. und 20. Jahrhundert anläßlich des 125jährigen Bestehens des Deutsche Vereins zur Erforschung Palästinas, (ADPV, 34).Wiesbaden, Harrassowitz, 2006, pp. 250-264.

${ }^{23}$ A. Glock. "Archaeology as Cultural Survival: The Future of the Palestinian Past". Journal of Palestine Studies, vol. 23, 1994, pp. 70-84; G. Ziadeh-Seely. "An Archaeology of Palestine: Mourning a Dream", en P.L. Kohl, M. Kozelsky y N. Ben-Yehuda, (editores). Selective Remembrances: Archaeology in the Construction, Commemoration, and Consecration of National Pasts. Chicago; The University of Chicago Press, 2007, pp. 326-345; H. Taha. "The Current State of Archaeology in Palestine".Present Pasts, vol. 2, 2010, pp. 16-25; E. Pfoh."Some Reflections on the Politics of Ancient History, Archaeological Practice and Nation-Building in Israel/Palestine", en E. Pfoh y K.W. Whitelam, (editores).The Politics of Israel's Past: The Bible, Archaeology and Nation-Building, (SWBA, 2/8). Sheffield, Sheffield Phoenix Press, 2013, pp. 1-17, esp. pp. 11-15.
} 


\section{El pasado de Palestina en disputa: Arqueología y religión en el conflicto palestino-israelí}

se ha renovado y existe un programa de educación de grado (B.A.) en arqueología en el ahora llamado Departamento de Historia y Arqueología de dicha universidad ${ }^{24}$.

Del lado occidental de la cuestión (Europa, EE.UU., Israel), la ya anticuada arqueología bíblica intentó modernizarse, especialmente durante las últimas tres décadas, apelando a nuevos métodos y horizontes de investigación, teniendo en cuenta en particular los debates y desarrollos historiográficos de la década de los '90. No obstante, dicha new biblical archaeology-como se la conoce, para diferenciarla de la arqueología bíblica del período 1920-1970- representa, en rigor, más una actualización de nombre que un cambio real de epistemología histórica. La matriz religiosa, o mejor dicho confesional, especialmente en el ámbito norteamericano, sigue dictando en gran medida la investigación y los modos de "reconstruir" la historia de Israel25. En efecto, en el ámbito de los estudios bíblicos donde dicha nueva arqueología bíblica tiene su mayor expresión-, la "historia de Israel" continúa siendo el marco general de investigación sobre el pasado de la Palestina antigua, tanto desde enfoques explícitamente conservadores ${ }^{26}$ como desde aquellos un tanto más críticos ${ }^{27}$.

Como contraparte de estos desarrollos preponderantes en la academia occidental, debe mencionarse la reciente constitución del Palestine's History and Heritage Project, iniciado en 2014 y dirigido por Thomas L. Thompson, Ingrid Hjelm y Mahmoud Issa de la Facultad de Teología de la Universidad de Copenhague en Dinamarca. La organización de este proyecto colectivo contempla la publicación de una historia general y comprensiva de Palestina, desde tiempos prehistóricos hasta la actualidad, en varios volúmenes y a cargo de diferentes especialistas internacionales, incluyendo entre ellos a investigadores israelíes y palestinos. Dicho esfuerzo plantea, en verdad, una versión alternativa sobre la manera en que se ha narrado tradicionalmente la historia antigua de Palestina desde Occidente, vale decir, con una preponderancia de la historia de Israel y del registro bíblico por sobre las diferentes realidades históricas que constituyen el pasado de la región, realidades que pueden ser recuperadas de mejor manera a partir de aportes no confesionales ni políticos de la arqueología, la geografía, la antropología y los

\footnotetext{
${ }^{24}$ Véase la página web<http://www.birzeit.edu/departments/history-archaeology> [Consulta: $27 / 06 / 2016]$.

25 Cf. la reciente síntesis de E.H. Cline.Biblical Archaeology: A Very Short Introduction.Oxford, Oxford UniversityPress, 2009; y también los ensayos reunidos en T.E. Levy, (editor).Historical Biblical Archaeology and the Future: The New Pragmatism.Londres, Equinox, 2010.Para una crítica, aunque algo moderada, y una reconsideración de la "nueva arqueología bíblica”, véase P.R. Davies. "A New 'Biblical Archaeology'”, en I. Hjelm y T.L. Thompson, (editores).Biblical Interpretation beyond Historicity: Changing Perspectives 7, (CIS).Londres, Routledge, 2016, pp. 15-28.

${ }^{26}$ Véase, por ejemplo, B.T. Arnold y R.S. Hess, (editores).Ancient Israel's History: An Introduction to Issues and Sources.Grand Rapids, Baker Academic, 2014.

27 Véase, por ejemplo y muy recientemente, C. Frevel. Geschichte Israels, (Kohlhammer Studienbücher Theologie). Stuttgart, Kohlhammer, 2016; y E.A. Knauf y P. Guillaume.A History of Biblical Israel: The Fate of the Tribes and Kingdoms from Merenptah to Bar Kochba, (WANEM).Sheffield, Equinox, 2016.
} 


\section{Emanuel Pfoh}

estudios culturales, para dar cuenta de los desarrollos locales e ininterrumpidos durante más de cinco milenios de historia ${ }^{28}$.

\section{Consideraciones finales}

El repaso general y sintético de los temas expuestos aquí tiene como principal objetivo generar una actitud crítica y reflexiva frente a los usos políticos del pasado, tanto del pasado como producto académico e historiográfico como del pasado imaginado colectivamente en la sociedad por parte de distintos actores. La limpieza étnica de Palestina, producida por los enfrentamientos bélicos de 1947 y por la creación del Estado de Israel en 1948, ha sido estudiada y expuesta por varios especialistas, entre otros, recientemente y de manera contundente, por Ilan Pappe ${ }^{29}$. Sin embargo, también deberíamos evitar que la limpieza cultural de Palestina, de sus memorias y tradiciones, siga produciéndose más de seis décadas después de 1948, no sólo para empoderar y concientizar a la sociedad palestina de su rico pasado histórico en la región, sino también porque el patrimonio cultural y arqueológico de Palestina pertenece a todos-judíos, cristianos y musulmanes- y no exclusivamente a sectores nacionales o étnicos particulares. Escribir una historia integral de Palestina, precisamente, debe atender a esta cuestión para poder superar así, entre otras cosas, la mirada sesgada que produce una racionalización del relato bíblico como principal fuente del pasado más antiguo de la región ${ }^{30}$.

Es menester aclarar, por cierto, que desde los años '70 en adelante la visión nacionalista-religiosa en el Estado de Israel, que vinculaba mandatos bíblicos con políticas de Estado con respecto a la población palestina y la hegemonía judía en el territorio, ha sido en general minoritaria en la sociedad israelí31. Sin embargo, si ampliamos nuestra perspectiva a los más de cien años de conformación del llamado conflicto palestino-israelí, podemos observar en términos generales, y con mínimas excepciones, que la falta de integración con la población local por parte de los primeros colonos judíos provenientes de Europa, y por quienes los siguieron, fue una práctica constante en el movimiento sionista. Ya sea desde un punto de vista secular como desde uno religioso, la población palestina nunca fue en realidad pensada como parte integral del Estado de Israel ${ }^{32}$. Asimismo, no escapa a la ironía

\footnotetext{
${ }^{28}$ Véase más información en <http://teol.ku.dk/pahh/english/> [Consulta: 27/06/2016].

${ }^{29}$ I. Pappe. La limpieza étnica de Palestina. Barcelona, Crítica, 2008 [edición original en inglés, 2006]. 30 Cf. T.L. Thompson. "Palestine's Pre-Islamic History and Cultural Heritage: A Proposal for Palestinian High-School Curriculum Revision". Holy Land Studies, vol. 12, 2013, pp. 207-233; K.W. Whitelam. "Shaping the History of Palestine: Nationalism and Exclusivity", en E. Pfoh y K.W. Whitelam, (editores).The Politics of Israel's Past: The Bible, Archaeology and Nation-Building, (SWBA, 2/8).Sheffield, Sheffield Phoenix Press, 2013, pp. 183-211.

${ }^{31}$ En general, sobre la sociedad israelí, cf. el estudio de B. Kimmerling. The Invention and Decline of Israeliness: State, Society, and the Military. Berkeley, University of California Press, 2001.

32 Cf. N. Masalha. Expulsion of the Palestinians: The Concept of 'Transfer' in Zionist Political Thought, 1882-1948.Washington, Institute for Palestine Studies, 1992; I. Pappe. "The Bible in the Service of Zionism: 'We do not believe in God, but he nonetheless promised us Palestine'”, en I. Hjelm y T.L. Thompson, (editores).History, Archaeology and the Bible Forty Years after "Historicity": Changing
} 


\section{El pasado de Palestina en disputa: Arqueología y religión en el conflicto palestino-israelí}

el hecho de que la colonización judía y la progresiva marginalización de la población local tuvieran como resultado la aceleración del proceso de conformación nacional de los palestinos, proceso cuyo origen puede rastrearse hasta fines del siglo XIX con la inminente desarticulación del imperio otomano ${ }^{33}$.

Cabría señalar también que la apelación al pasado bíblico como elemento de legitimación histórica de la política israelí desde la creación del Estado ya no puede ser sostenida a partir de criterios formalmente históricos. En las últimas décadas, la historiografía moderna sobre el llamado "antiguo Israel" ha refutado de manera contundente gran parte de la asumida historicidad del relato bíblico. Los patriarcas Abraham, Isaac y Jacob ya no pueden ser comprendidos como personajes realmente históricos. Del éxodo de los antiguos israelitas desde Egipto, no existen pruebas históricas o indicios convincentes. Inclusive el gran reino de David y Salomón no puede ser considerado histórico, a partir de lo que la arqueología ha revelado en las últimas décadas. En suma, la conclusión que puede obtenerse de este progreso historiográfico es, en primer lugar, que la Biblia no puede ser leída de manera literal o en un sentido moderno e histórico, sino que debe interpretarse con mucho cuidado, como un conjunto complejo de alegorías mítico-teológicas que apela a eventos del pasado pero sin que ello implique la historicidad necesaria de estos eventos, y en segundo lugar, que la historia más antigua de la región debe escribirse apelando a las fuentes primarias (arqueológicas, epigráficas y textuales) antes que a partir de una racionalización de los relatos bíblicos o siguiendo políticas nacionalistas modernas ${ }^{34}$.

Desde su instauración como Estado judío, antes que como Estado de todos sus habitantes, y sostenido en una apelación a una relación histórica del pueblo israelita con Palestina, el Estado de Israel se ha constituido en una comunidad civil y política en cierta medida exclusiva, a la cual no pueden pertenecer de manera plena y sin inconvenientes todas las personas que habitan su territorio o que han nacido en él, sino solamente una mayoría etnorreligiosa, mayoría que comenzó siendo una minoría y que alcanzó aquel grado a partir de un proceso de progresivo desplazamiento territorial de la población palestina ${ }^{35}$. Más aún, ese Estado extiende su potencial pertenencia a individuos extraterritoriales, vale decir, a ciudadanos de otras naciones, nacidos en otros países, pero que profesan la fe judía o son descendientes de quienes la profesaban, mientras que niega a otros individuos

Perspectives 6, (CIS). Londres, Routledge, 2016, pp. 205-217; pero cf. también Arav. “Archaeology in the Service of Ideology", Op. Cit., pp. 100-102.

${ }^{33} \mathrm{Cf}$. Y. Porath, "The Political Awakening of the Palestinian Arabs and Their Leadership towards the End of the Ottoman Period", en M. Ma'oz, (editor).Studies on Palestine during the Ottoman Period.Jerusalén, TheMagness Press, 1975, pp. 351-381; Khalidi.PalestinianIdentity..., Op. Cit., pp. 93117.

${ }^{34} \mathrm{Al}$ respecto de estos desarrollos, véase T.L. Thompson. Biblical Narrative and Palestine's History: Changing Perspectives 2, (CIS).Sheffield, Equinox, 2013; y N.P. Lemche. Biblical Studies and the Failure of History: Changing Perspectives 3, (CIS). Sheffield, Equinox, 2013.

35Véase O. Yiftachel. Ethnocracy: Land and Identity Politics in Israel/Palestine.Philadelphia, University of Pennsylvania Press, 2006, pp. 101-130; también Sand. The Invention of the Jewish People..., Op. Cit., pp. 250-313. 


\section{Emanuel Pfoh}

nacidos en Nablus, Ramallah o Jerusalén y de fe cristiana o musulmana la posibilidad de ser ciudadanos en iguales condiciones de derecho en dicho Estado. Esta desigualdad etnojurídica no puede ser mantenida en el Estado israelí sin esperarse que genere crónicamente crisis y conflicto interno. Sin ser categóricos, y sin descartar en absoluto la posibilidad de que exista en el futuro un Estado Palestino soberano y autónomo sobre su territorio, podríamos postular también que, tal vez, la clave de la resolución del llamado conflicto palestino-israelí radique, no en la separación material a través de muros, no en la exclusión territorial ni en el control físico y humillante de la población palestina confinada espacial y simbólicamente ${ }^{36}$ medidas sostenidas a partir de instancias atribuidas a razones de seguridad nacional pero también fundadas, en cierto modo, en apelaciones a un pasado bíblico acerca de la propiedad de la tierra-, sino más bien en la valiente y correcta decisión política de abrir el Estado de Israel a todos sus ciudadanos, no sólo aquellos que se identifican como judíos, y crear de esa manera una sociedad israelí verdaderamente democrática e inclusiva.

\section{Bibliografía}

A. Glock. "Archaeology as Cultural Survival: The Future of the Palestinian Past". Journal of Palestine Studies, vol. 23, 1994, pp. 70-84.

B. Mershen y U. Hübner. "Tawfīq Canaan and His Contribution to the Ethnography of Palestine", en U. Hübner (editor).Palaestina exploranda. Studien zur Erforschung Palästinas im 19. und 20. Jahrhundert anläßlich des 125jährigen Bestehens des Deutsche Vereins zur Erforschung Palästinas, (ADPV, 34).Wiesbaden, Harrassowitz, 2006, pp. 250-264.

B. Ra'ad. Hidden Histories: Palestine and the Eastern Mediterranean. Londres, Pluto Press, 2010.

B. Scarcia Amoretti. "La Palestine musulmane", en A. Giardina, M. Liverani y B. Scarcia Amoretti. La Palestine: Histoire d'une terre, (Comprendre le Moyen Orient). París, L'Harmattan, 1990.

B.T. Arnold y R.S. Hess, (editores).Ancient Israel's History: An Introduction to Issues and Sources.Grand Rapids, Baker Academic, 2014.

C. Frevel. Geschichte Israels, (Kohlhammer Studienbücher Theologie). Stuttgart, Kohlhammer, 2016; y E.A. Knauf y P. Guillaume.A History of Biblical Israel: The Fate of the Tribes and Kingdoms from Merenptah to Bar Kochba, (WANEM).Sheffield, Equinox, 2016.

C. Tyerman. God's War: A New History of the Crusades. Londres, Penguin Books, 2007.

D. Gavish. A Survey of Palestine under the British Mandate, 1920-1948. Londres, Routledge Curzon, 2005.

${ }^{36}$ Véase, en particular, E. Weizman. Hollow Land: Israel's Architecture of Occupation.Londres, Verso Books, 2007. Cf. también Yiftachel, Ethnocracy..., Op. Cit. 


\section{El pasado de Palestina en disputa: Arqueología y religión en el conflicto palestino-israelí}

D. Trimbur y R. Aaronsohn, (editores).De Bonaparte à Balfour: La France, l'Europe occidentale et la Palestine, 1799-1917. París, CNRS Éditions, 2008 [2001].

Decline of Israeliness: State, Society, and the Military. Berkeley, University of California Press, 2001.

E. Pfoh. "Geografías imaginadas, práctica arqueológica y construcción nacional en Israel/Palestina”. Cuadernos de Antropología Social, vol. 39, 2014, pp. 39-62.

E. Pfoh."Some Reflections on the Politics of Ancient History, Archaeological Practice and Nation-Building in Israel/Palestine", en E. Pfoh y K.W. Whitelam, (editores).The Politics of Israel's Past: The Bible, Archaeology and Nation-Building, (SWBA, 2/8). Sheffield, Sheffield Phoenix Press, 2013, pp. 1-17.

E. Weizman. Hollow Land: Israel's Architecture of Occupation.Londres, Verso Books, 2007.

E.H. Cline.Biblical Archaeology: A Very Short Introduction.Oxford, Oxford UniversityPress, 2009; y también los ensayos reunidos en T.E. Levy, (editor).Historical Biblical Archaeology and the Future: The New Pragmatism.Londres, Equinox, 2010.

E.H. Tejirian y R. Spector Simon. Conflict, Conquest, and Conversion: Two Thousand Years of Christian Missions in the Middle East. Nueva York, Columbia University Press, 2012.

Finkelstein.The Archaeology of the Israelite Settlement. Jerusalén, Israel Exploration Society, 1988, esp. pp. 34-117.

G. Biger. The Boundaries of Modern Palestine, 1840-1947.Londres, RoutledgeCurzon, 2004.

G. Krämer. Historia de Palestina: Desde la conquista otomana hasta la fundación del Estado de Israel. Madrid, Siglo XXI, 2006 [edición original en alemán, 2002].

G. Piterberg. The Returns of Zionism: Myth, Politics and Scholarship in Israel. Londres, Verso Books, 2008.

G. Ziadeh-Seely. "An Archaeology of Palestine: Mourning a Dream”, en P.L. Kohl, M. Kozelsky y N. Ben-Yehuda, (editores). Selective Remembrances: Archaeology in the Construction, Commemoration, and Consecration of National Pasts. Chicago; The University of Chicago Press, 2007, pp. 326-345.

H. Donner. Pilgefahrt ins Heilige Land: Die ältesten Berichte christlicher Palästinapilger (4.-7. Jh.). Stuttgart, Verlag Katholisches Bibelwerk 2002.

H. Goren. "Scientific Organizations as Agents of Change: The Palestine Exploration Fund, the Deutsche Verein zur Erforschung Palästinas and Nineteenth-Century Palestine". Journal of Historical Geography, vol. 27, 2001.

H. Goren. "Zieht und erforscht das Land«. Die deutsche Palästinaforschung im 19. Jahrhundert. Gotinga, Wallstein, 2003; idem.Dead Sea Level: Science, Exploration and Imperial Interests in the Near East. Londres, I.B. Tauris, 2011.

H. Taha. "The Current State of Archaeology in Palestine".Present Pasts, vol. 2, 2010, pp. 16-25. 


\section{Emanuel Pfoh}

I. Pappe. "The Bible in the Service of Zionism: 'We do not believe in God, but he nonetheless promised us Palestine'”, en I. Hjelm y T.L. Thompson, (editores).History, Archaeology and the Bible Forty Years after "Historicity": Changing Perspectives 6, (CIS). Londres, Routledge, 2016, pp. 205-217.

I. Pappe. La limpieza étnica de Palestina. Barcelona, Crítica, 2008 [edición original en inglés, 2006].

J. Barr. A Line in the Sand: Britain, France and the Struggle that Shaped the Middle East. Londres, Simon \&Schuster, 2011.

J.J. Moscrop. Measuring Jerusalem: The Palestine Exploration Fund and British Interest in the Holy Land.Leicester, Leicester University Press, 2000.

K.W. Whitelam. "Shaping the History of Palestine: Nationalism and Exclusivity", en E. Pfoh y K.W. Whitelam, (editores).The Politics of Israel's Past: The Bible, Archaeology and Nation-Building, (SWBA, 2/8).Sheffield, Sheffield Phoenix Press, 2013, pp. 183-211.

M. Azaryahu y A. Golan. "(Re)Naming the Landscape: The Formation of the Hebrew Map of Israel 1949-1960".Journal of Historical Geography, vol. 27, 2001, pp. 178-195. M. Benvenisti. Sacred Landscape: The Buried History of the Holy Land since 1948.Berkeley, University of California Press, 2000.

M. Feige. "Recovering Authenticity: West-Bank Settlers and the Second Stage of National Archaeology", en P.L. Kohl, M. Kozelsky y N. Ben-Yehuda, (editores).Selective Remembrances: Archaeology in the Construction, Commemoration, and Consecration of National Pasts. Chicago, University of Chicago Press, 2007, pp. 277-298.

M. Hawari. "The Citadel of Jerusalem: A Case Study in the Appropriation of Archaeology in Palestine". Present Pasts, vol. 2, 2010, pp. 89-95.

N. Abu el-Haj.Facts on the Ground: Archaeological Practice and Territorial SelfFashioning in Israeli Society. Chicago, The University of Chicago Press, 2001.

N. Masalha. Expulsion of the Palestinians: The Concept of 'Transfer' in Zionist Political Thought, 1882-1948.Washington, Institute for Palestine Studies, 1992.

N. Masalha. Imperial Israel and the Palestinians: The Politics of Expansion. Londres, Pluto Press, 2000, pp. 105-162.

N.A. Silberman. Digging for God and Country: Exploration, Archaeology, and the Secret Struggle for the Holy Land, 1799-1917.Nueva York, Doubleday, 1982.

N.P. Lemche. "History as an Argument for Land Possession", en E. Pfoh y K.W. Whitelam, (editores).The Politics of Israel's Past: The Bible, Archaeology and NationBuilding, (SWBA, 2/8). Sheffield, Sheffield Phoenix Press, 2013, pp. 102-119.

N.P. Lemche. Biblical Studies and the Failure of History: Changing Perspectives 3, (CIS). Sheffield, Equinox, 2013.

O. Yiftachel. Ethnocracy: Land and Identity Politics in Israel/Palestine.Philadelphia, University of Pennsylvania Press, 2006, pp. 101-130.

P.R. Davies."A New 'Biblical Archaeology'”, en I. Hjelm y T.L. Thompson, (editores).Biblical Interpretation beyond Historicity: Changing Perspectives 7, (CIS).Londres, Routledge, 2016, pp. 15-28. 


\section{El pasado de Palestina en disputa: Arqueología y religión en el conflicto palestino-israelí}

P.R.S. Moorey.A Century of Biblical Archaeology. Cambridge, Lutterworth Press, 1991.

R. Arav. "Archaeology in the Service of Ideology in Israel", en L.J. Greenspon y R.A. Simkins (eds.), "A Land Flowing with Milk and Honey": Visions of Israel from Biblical to Modern Times.Omaha, Creighton University Press, 2001, pp. 95-104.

R. Greenberg. "Extreme Exposure: Archaeology in Jerusalem, 19672007".Conservation and Management of Archaeological Sites, vol. 11, 2009, pp. 262281.

R. Kark. "Land-God-Man: Concepts of Land Ownership in Traditional Cultures in Eretz-Israel", en A.R.H. Baker y G. Biger, (editores).Ideology and Landscape in Historical Perspective. Cambridge, Cambridge University Press, 1992, pp. 63-82.

R. Khalidi. Palestinian Identity: The Construction of Modern National Consciousness. Nueva York, Columbia University Press, 1997.

R.L. Wilken. The Land Called Holy: Palestine in Christian History \& Thought. New Haven, Yale University Press, 1992.

S. Lehmann-Brauns. Jerusalem sehen. Reiseberichte der 12. und 15. Jahrhunderts als empirische Anleitung zur geistigen Pilgefahrt, (Berliner Kulturwissenschaft, 9). Freiburg im Breisgau, Rombach Verlag, 2010.

S. Sand. The Invention of the Jewish People. Londres, Verso Books.

S. Sand. The Invention of the Land of Israel: From Holy Land to Homeland. Londres, Verso Books, 2012.

T.L. Thompson. "Palestine's Pre-Islamic History and Cultural Heritage: A Proposal for Palestinian High-School Curriculum Revision”. Holy Land Studies, vol. 12, 2013, pp. 207-233.

T.L. Thompson. Biblical Narrative and Palestine's History: Changing Perspectives 2, (CIS).Sheffield, Equinox, 2013.

T.L. Thompson. Early History of the Israelite Peoples: From the Written and Archaeological Sources, (SHANE, 4). Leiden, E.J. Brill, 1992.

V. Türck. Christliche Pilgerfahrten nach Jerusalem im früheren Mittelalter im Spiegel der Pilgerbericht, (ADPV, 40).Wiesbaden, Harrassowitz, 2011.

Y. Porath, "The Political Awakening of the Palestinian Arabs and Their Leadership towards the End of the Ottoman Period", en M. Ma'oz, (editor).Studies on Palestine during the Ottoman Period.Jerusalén, TheMagness Press, 1975, pp. 351-381.

Recibido: 08/09/2016

Evaluado: 27/10/2016

Versión Final: 24/11/2016 